\title{
A processural consent methodology with people diagnosed with dementia
} Tessa Hughes and Maria Castro Romero

\author{
Abstract \\ Purpose - The purpose of this paper is to develop an approach within the guidance of the \\ Mental Capacity Act (2005) to meaningfully include people diagnosed with dementia (PDwD) \\ in research endeavours. \\ Design/methodology/approach - As part of a broader study of self-authored narratives of \\ care experiences, PDwD were involved in the development and implementation of a process \\ method of consent, in which consent conversations were contextual, responsive and \\ ongoing, and were audited with the use of field diaries. \\ Findings - Working within people's relational contexts (i.e. care staff and family), eight \\ participants with a range of dementia diagnoses and care needs made and verbally \\ communicated research-related decisions. \\ A desire to participate was consistently conveyed across research encounters, regardless of \\ the extent of memory problems. Participants also demonstrated keen awareness of the links \\ between memory problems, rights and inclusion, alongside a sense of personal identity and \\ the capacities to clearly communicate this. \\ Research limitations/implications - A process model of consent encouraged formal reflection \\ upon ethical and pragmatic complexities, and is relevant to persons diagnosed with \\ dementia making both care- and research-related decisions. Further work is needed to \\ include people with a broader range of communication support needs. \\ Originality/value - This research demonstrates substantial possibilities for eliciting and \\ responding to the views of people with dementia diagnoses (previously excluded from \\ research). Results open opportunities for genuine long-term research and care partnerships \\ with PDwD for practice, service and policy development. \\ Keywords Dementia, Mental capacity, Participation, Narrative, Inclusion, Person-centred \\ care
}

\section{Introduction}

Methods to elicit older people's experiences of health and social care remain limited, especially for those with higher support needs (Katz et al., 2011), despite explicit statutory requirements for user participation (e.g. Department of Health, 2001, 2012). There are structural flaws in health and social care regulatory systems, with surveys derived from professionals' and services' perspectives and focusing on minimum standards rather than user experience (McCormick et al., 2009). Such evaluations therefore mean little to people who access services. Furthermore, there is limited representation of older service users' perspectives in the literature, which in the UK can be contextualised within discourses and elder cohorts' beliefs (particularly for those born before 1948) related to the right to speak, gratitude for universally free health and social care, low expectations and fear of service cuts if provision is criticised (Castro Romero, 2016; McCormick et al., 2009).

The experiences of people diagnosed with dementia (PDwD[1]) have been particularly seldom heard as part of the policy and service development process (Robson et al., 2008). Despite examples of good practice (e.g. Barnett, 2000; Keady and Williams, 2007), substantial barriers exist, predominantly professional assumptions that inclusion is prohibited by the effects of cognitive impairment and an assumed lack of capacity to be involved in decision making (Hernandez et al., 2010). Research methodologies that consider the experiences of PDwD as central (e.g. Clark-McGhee and Castro, 2013; Dupuis et al., 2012) are under-represented in the literature, relative to those that prioritise professional or other stakeholder agendas. This poor track-record of inclusion is concerning not only on moral grounds, in that it subjugates the voices of an already marginalised group, but pragmatic ones, as we currently lack the knowledge to develop services that respond to the self- 
perceived needs of people who access them. Indeed, Fox et al. (2013) outline the potential harm that could be caused by increasing dementia awareness and screening in an ageing population, in the absence of a strategic approach to research to determine where it will provide tangible benefits. Amongst other things, this requires increased knowledge about what dementia care services are meaningful for PDwD.

\section{Mental capacity, consent and decision making}

In practice, conceptual clarity, pragmatism and creativity are necessary to ethically elicit and respond to the perspectives of PDWD with a range of cognitive and communicative abilities. Our approach draws upon and expands the ethical framework of the Mental Capacity Act (MCA) (HMSO, 2005). "Mental capacity" is broadly defined as the ability to make decisions for oneself, and in England and Wales the MCA provides a statutory framework to protect those over 16 years old who may not be able to do so. A person's capacity may be affected by illness, injury or congenital disability affecting cognitive functioning, and the context and nature of the decision to be made (HMSO, 2005). Therefore, capacity is fluid, and assessment should be time- and decision-specific, enabling the person to understand, retain, weigh-up and communicate the relevant information and decision made to the best of their ability.

This study focuses on facilitating the inclusion of those with cognitive impairments who are nonetheless able to make decisions for themselves. The MCA (HMSO, 2005) requires researchers to decide whether to include people without the capacity to consent to participation according to whether the research relates to the condition that affects capacity, and whether the research could be undertaken as effectively with people who do have the capacity to consent. As argued below, whilst a dementia diagnosis can be associated with reduced capacity, it is not necessarily so, i.e. dementia research can be undertaken with those with capacity to consent. Our concern was to assess and, where possible, enable inclusion within the context of cognitive impairment, as opposed to include those unable to consent for themselves. It is worth noting however, that for studies where people without capacity to consent are included, the MCA also outlines particular conditions under which research can still proceed. This includes with the support of a personal consultee, i.e. someone close to the person, in an unpaid role, to advise on whether she or he would want to be involved, and research ethics approval from an appropriate body (Department for Constitutional Affairs, 2007).

Dementia diagnostic processes have been critiqued with regards to reliability and validity; decontextualisation of the person's cognitive and communicative functioning; and overreliance on neuropsychological test batteries (Fox et al., 2013; Sabat and Gladstone, 2010). Nonetheless, a dementia diagnosis is generally associated with decline in cognitive functions such as attention, memory and communication (Alzheimer's Society, 2012). Historically, it has been mistakenly assumed that these factors strip the individual of their mental capacity and preclude involvement in therapy, research, and even care-related decision making (e.g. Cohen and Eisdorfer, 1986).

Despite the primacy of the "person-centred" care discourse in current UK dementia policy and guidance (Department of Health, 2012; National Institute for Health and Care Excellence, 2011), in practice, a "biomedical-legal" model often links dementia diagnoses and related cognitive impairment to a loss of citizenship. This restricts the definition of capacity - and arguably what it means to be human - to the capacity to think with selfawareness and rationality (Behuniak, 2010). PDwD continue to be, by virtue of the diagnosis, automatically excluded from research, and from decisions about their own lives (Hernandez et al., 2010); practices that contravene the requirements of capacity assessment as outlined under the MCA (HMSO, 2005). Whilst working with people with cognitive difficulties involves serious ethical considerations, as outlined below, previous research has 
successfully elicited emotional memories from people with moderate-to-severe cognitive impairment (e.g. Mills, 1997) and found interpretative and2000; Castro and Clark-McGhee, 2014).

Iphofen (2011) outlines the ethics and fluidity of consent in research, both generally, and particularly for those with impairments that may affect capacity. By thoughtfully questioning who is consenting to what, and how, Iphofen suggests that researchers can devise appropriate (although not necessarily error-free) methodologies to balance inclusion with protection of participants in a particular context. For example, whilst for some high-risk research endeavours signed and witnessed consent may be appropriate, in much qualitative research a dialogue in which information given is concurrent with audiotaped verbal consent is more congruent with the research process. Indeed, qualitative researchers have argued that giving and obtaining consent to participation is an ongoing dialogue between researcher and researched (e.g. Elliott, 2005).

Further, regardless of capacity, consent is viewed as a process, and participants are active agents, potentially transformed by, and transforming, the research process, rather than serving merely as sources of data (Smythe and Murray, 2000).

Person-centred models aim to broaden the conceptual basis and care practices related to dementia, yet there is a poor record of operational definitions and evidence for implementation in the literature (Epp, 2003). An alternative to both poorly defined "personcentred" and restrictive "biomedical-legal" models is a broader construction of capacity and inclusion to encompass compassion, balance rights with protection (Behuniak, 2010), and attend to the relational processes involved in decision making. This paper reports on the development of such a process of consent in regard to participation in research, in which the perspectives of PDwD were elicited to inform recommendations for dementia care.

\section{Research aims}

Based on a doctoral study using qualitative narrative methodology, this paper focuses on the ethics and methodology of recruiting and co-researching with PDwD.

Broadly, the research aimed to elicit and analyse narrative accounts by PDwD regarding their experiences of health and social care. The aim was to understand how individual perspectives relate to service provision - and the policy frameworks informing this in the UK at present - in order to make recommendations regarding what PDwD value. Alongside this, we aimed to contribute to the development of alternative methodologies in dementia care and research to facilitate the participation of PDwD.

\section{Method}

The impact of each individual's cognitive ability, and the "negotiated social relations" (Wilkinson, 2002, p. 16) between researcher and researched that ensure ethical participation, are two key considerations in research with PDwD. Our methodology drew from examples of good practice in the literature (e.g. Allan, 2001; Dewing, 2007) and feedback from our consultants. Key methodological processes are presented here alongside reflections on the successes and challenges encountered.

\section{Consultation with $P D w D$}

To consult with PDwD, we developed links with an inner-London branch of the Alzheimer's Society, a leading charity for people affected by dementia. A consultation group of five PDwD informally self-selected from a social support group with approximately 25 attendees (including carers). 
Together, we developed the "conversational prompts" for the research encounters, and they advised on the procedures to ensure that the research was relevant and acceptable to PDwD.

During the process of consultation, carers often attempted to protect their relatives from what they perceived as the stress of communication, with interventions such as "he won't understand that". They also frequently expressed the view that it is carers themselves who hold the information about what is helpful in the care of their relatives. Whilst we acknowledged that family caregivers often negotiate with, and are required to become experts in, care providers and procedures (Ayres, 2000), we maintained a focus on talking with PDwD directly in order to fulfil our research aims. The resultant feedback from PDwD was clear; for example, in advising that a researcher should talk them through the information sheets, and in expressing the value of unstructured, responsive conversations and their own ability to convey their views, as illustrated in the below quotes from our consultants:

Meet us where we're at on the day (Beth[2]).

Ask me; if it's reasonable I'll answer, if it's unreasonable, I'll tell you (Josiah).

\section{Overview of research procedures}

Based upon the consultants' advice, data collection comprised a combination of group conversations and one-to-one interviews, both of which have been successfully utilised in previous dementia research (e.g. Hernandez et al., 2010; Hubbard et al., 2003).

Participants were recruited to either the group or interviews. Both the one-to-one and group conversations comprised unstructured conversations, following a uniform opening question suggested by our consultants to elicit the stories of participants in relation to professional dementia care:

Can you tell me about a time when you have received care from staff?

The duration and pacing of the encounters were dictated by the participants and informed by each interaction, which assumed its own pattern as the researcher followed and prompted the participants' stories of care.

In total, three group sessions were held, with the same four participants at each, and a fifth participant in the first group, with a duration of 39-45 minutes per group. One-to-one interviews with an additional three participants were conducted, ranging in length from 28 to 58 minutes.

\section{Recruitment}

Following ethical approval from our university's research ethics committee, PDwD were recruited via an Integrated dementia Day Service (IDS) within an outer-London adult social care service, and the Alzheimer's Society branch which hosted the consultation.

Staff at these recruitment sites, with the consent of participating service leads, were asked to identify people who met the inclusion criteria, which initiated the process-consent method outlined below.

Inclusion criteria

1. participants were required to be in receipt of dementia care services as the person with the diagnosis (or potential diagnosis), i.e. not as a carer; and

2. an ability to express oneself in English was required to enable the researcher, an Englishspeaker, to undertake a thorough narrative analysis of the transcripts. 
Particular diagnoses, types of care experience or levels of cognitive ability were not prerequisites for inclusion.

\begin{abstract}
Informed consent
The processes outlined here are congruent with the aims of the MCA (HMSO, 2005) to assist people in making their own decisions. McKeown et al. (2010) argue that traditional consent approaches, such as proxy consent and cognitive-competency-based approaches, do not respect the perspectives of PDwD and exclude people with cognitive impairment. With this in mind (and congruent with the centrality of PDwD in this research), our primary ethical concern was for each participant to be central to determining their own involvement with this research, within appropriate relational contexts. In acknowledging the complexity of balancing rights (e.g. the right to give one's opinion or to inclusion) with protection (e.g. from coercion and misuse of professional power by the researcher or loss of privacy), a process of consent was developed. Whilst within the terms of the MCA this did not require a formal role for personal consultees, as those without the capacity to consent were not included in the research (Department for Constitutional Affairs, 2007), we instead drew upon our skills as clinicians to understand and work within the relational contexts of formal and informal care to continuously assess and enable capacity and inclusion for each potential participant. The recognition that both decision making in general, and the cognitive and functional impairments associated with dementia, are contextual, guided the development of consent as a process that crosses contexts (e.g. time and method of information giving). This was evidenced, as per Cowdell's (2008) recommendation, by a field-diary maintained by the lead researcher.
\end{abstract}

This provided an "audit trail" of decisions made and actions taken, and a basis for reflective research-team discussions. Consent to participate throughout the research process was assessed and recorded, as outlined in Box 1.

Box 1: Overview of the process-consent model

1. Establishing basis for consent: staff at the recruitment sites identify people who meet the inclusion criteria. For those who usually involve a carer, friend or relative in their decision making, provide the carer with an information sheet and ask for written or verbal indication as to whether they know of any reason why their relative would object to, or be distressed by, being approached to discuss or participate in research. Where no carer is usually involved in decision making with the PDWD, researchers work with staff to understand the usual ways in which the person would communicate consent or non-consent, and record evidence of such. Staff approach potential participant and seek verbal consent for researchers to introduce themselves and the research. If the above processes indicate that it is acceptable, researchers meet each potential participant and invite them into initial conversation about the research. 2. Initial consent conversation: an unhurried consent meeting between researchers and potential participants. Share with each potential participant an accessible illustrative information sheet (piloted with consultants). Explain information verbally, and elicit consent to participation verbally and behaviourally, reflexively checking understanding and consent. Researchers maintain field notes and discuss observations with staff and carers to contribute to the information available to aid the informed-consent process. (Note: this is an alternative to seeking written consent, which in the context of dementia may create anxiety as when people may remember signing an official form, but not recall why).

3. Ongoing consent monitoring: assessment of the individual's choice to continue participation. Includes the researchers' monitoring of behaviour and verbal utterances to assess frustration, tiredness, anxiety, etc., and asking both when these cues indicate distress and, at regular intervals in the research encounter, whether the individual continues to assent to participation and/or would like to reschedule. Request consent to use the data collected for analysis and write-up on completion of the interview. Participants, carers and staff provided with contact details for the researchers for discussion or queries regarding participation. 
4. Support: the process-consent method requires researchers' critical reflection and skills in their interactions with the person with dementia. In this research, this was supported by the researchers' clinical experience in a dementia context and supervision.

Source: Adapted from Dewing (2007)

\section{Participant demographics}

Demographic data are offered at a group level for methodological context (see Tables I and II). The aim of this data is primarily to indicate the range of diagnostic labels and (multiple) care needs of people included in the research. This was not a comparative study across dementia diagnoses or type of support received; rather, the focus was on considering the accounts of PDwD of professional dementia care. Demographics were gathered directly with participants, and, with their permission, in liaison with staff and carers.

Table I Participant demographics

\begin{tabular}{lll}
\hline Mean age & Gender & Ethnicity \\
80.5 years & Female $\left(\mathrm{n}^{1 / 43)}\right.$ & White-British $\left(\mathrm{n}^{1 / 46)}\right.$ \\
(range: $70-91$ years) & Male $\left(\mathrm{n}^{1 / 45)}\right.$ & White-other $\left(\mathrm{n}^{1 / 41)}\right.$ \\
& & Asian British-Pakistani $\left(\mathrm{n}^{1 / 4} \mathbf{4}\right)$
\end{tabular}

Table II Participant dementia diagnosis and related care provision

\begin{tabular}{|c|c|c|}
\hline Dementia diagnosis & Range of years since diagnosis & Dementia-related service \\
\hline $\begin{array}{l}\text { Alzheimer's disease }\left(n^{1 / 42}\right) \\
\text { Mild cognitive impairment }\left(n^{1 / 43)}\right. \\
\text { Dementia Lewy bodies }\left(n^{1 / 41)}\right. \\
\text { Parkinson's-related }\left(n^{1 / 41)}\right. \\
\text { Diagnosis undisclosed }\left(n^{1 / 41)}\right.\end{array}$ & 6 months -9 years & $\begin{array}{l}2-3 \text { days per week day-care, } \\
\text { social care provision }\left(n^{1 / 45)}\right. \\
\text { Sheltered housing }\left(n^{1 / 42)}\right. \\
\text { Memory clinic review, } \\
\text { usually biannually }\left(n^{1 / 48)}\right. \\
\text { Voluntary respite carer }\left(n^{1} 1 / 41\right) \\
\text { Care primarily by family }\left(n^{1 / 42)}\right. \\
\text { Personal care package - } \\
\text { social care }\left(n^{1 / 43)}\right. \\
\text { Voluntary sector social } \\
\text { support }\left(n^{1 / 43)}\right.\end{array}$ \\
\hline
\end{tabular}

Following guidance from the researchers regarding the broad inclusion criteria, frontline staff at the IDS were asked to identify four to six PDwD for a small group - a suitable sample size for detailed narrative analysis. Five out of the 60 people who accessed the IDS were identified as meeting the inclusion criteria, and all five consented to participate. At the Alzheimer's Society, where again we had requested a small sample size to enable detailed analysis, four people were identified as meeting the inclusion criteria by staff - selected from a population of approximately 500 service users (excluding carers) - and each consented to participate in the interviews. One potential participant experienced unexpected housing problems and subsequently declined to participate.

\section{Findings}

Staff advised that six of the eight participants usually made and verbally communicated carerelated decisions independently, and therefore could be reasonably expected to do the same regarding the research, with appropriate support. The remaining two participants usually consented to care decisions in discussion with their family carers. In accordance with the processes outlined in Box 1, their carers were approached first, and were provided with an information sheet. Both carers were clear that it was up to their relative whether they wanted to be involved, and recommended direct discussion with them. With the initial basis for 
consent established, we worked with staff and family to understand the usual ways in which the person would communicate consent or non-consent, and recorded evidence of such in the field diaries. In practice, all eight participants verbally consented, following the procedures outlined in Box 1.

Participants demonstrated understanding of the research by, for example, asking appropriate questions in response to information given, such as "Will my real name be in the report?", and by offering suggestions for appropriate pseudonyms. Consent was also recorded verbally on audio equipment at the beginning of each research encounter.

\section{Consent reliability}

It is interesting that authors of previous research (e.g. Helgesen et al., 2010) have stated that the perspectives of PDWD may be unreliable. It is our contention, supported by the research ethics literature (e.g. Iphofen, 2011), that anyone may change their mind about a decision made and, therefore, that process-consent methods are ethical alternatives to "one off" elicitations of consent for any research endeavour. In response to dementia-specific concerns regarding confusion and forgetfulness about what has been consented to, it was our experience that even for those with severe memory problems, those who, for example, did not recognise the researcher or recall details of a previous conversation, a consistent wish to participate was conveyed. While one man did not recall the previous two research conversations and letters received prior to the research encounter, he nevertheless consistently gave his consent afresh at each encounter, congruent with personal values that he expressed during each encounter about the importance of education, research knowledge and helping others.

\section{Consent validity}

There were occasions when people did lose track of the purpose of our research conversations, and this is where the clinical experience of the researchers - a trainee and a clinical psychologist, both with clinical experience in dementia care - was most important. To record and analyse conversation which a person is not aware they are sharing for research purposes is clearly unethical. As researchers, we made regular interventions to check if the topic was related to our endeavour. Sometimes seemingly unrelated topics were in fact related to it by the participant; for example, one man's narrative of his shipping career was linked to his current day-care services by the topic of standards of service and food. At other times, participants advised that their comments were unrelated, and so these were not interpreted within the research analysis. In addition, a flexible approach, such as nonintervention when people walked out of the room, enabled participants to take control of their participation. It was important that as researchers we were skilled in eliciting and responding sensitively to personal stories, and in reflexive decision making regarding verbal and behavioural cues to consent throughout our encounters.

\section{Capacity decisions and identity}

Participants were keenly aware of the links between memory problems, rights and inclusion. Professional discourses construct PDwD as "vulnerable adults" (Behuniak, 2010), allowing the capacity of PDwD to be questioned in practice without sound evidence of incapacity. As Boyle (2003) argues, the discourse of "vulnerability", particularly in contexts of professional care, constructs a negative, socially conferred identity associated with helplessness and powerlessness. The following exchange with the participant referred to as "Mick" in a research interview highlights the effects of this positioning:

Mick: But people that have got problems, there are people erm, using them abusing them. 
$[\ldots]$

Mick: I'm not getting the factualities of problems [...] nobody's coming to, to advise

me.

Lead Researcher: Oh, I see, so you don't feel like you're having things explained properly?

Mick: Yeah yeah yeah, and people are taking advantage of that, mean advantage.

Throughout our encounter Mick shifted between his apparently well-worn life story of independence and having made his own way, to a frightening position of losing control, with the legal loss of his citizenship via a friend having legal power of attorney over his financial affairs:

Mick: I'm forgetful, they aim to use the law against me, well you gave it, he had the power to use it, you know, it never gets round to the fact that the man has a problem, he wasn't aware of this [...] Yeah.

Lead Researcher: So, do you think that people are oMick: It's turned meW taking advantage?

Mick: Yes. Yeah. Now nobody's perfect, I'm not perfect. But it's turned me. I couldn't believe it [...] he nearly lost, he has three houses he nearly lost them [...] and I he was about to sell one as an [...] and it all died down, without this we'd have been very friendly. [...] and now and I ask for my money back he said "no"[...] And I wanted it to buy, buy things, and he wouldn't even give me the price of a loaf of bread [...] I mean that's the insult, that's an insult to me.

Mick affirms his personal identity by reinforcing his own capacity to resist attempts by others to push him around, whilst also recognising the strength of the legal discourses and their ability to alter his personae and the attendant concrete limitations imposed on his self[4].

Rather than speaking of himself as vulnerable, Mick has instead "turned" to become hard, talking angrily rather than fearfully. The narrative strength of Mick's selfhood provides evidence to support previous research which found that a sense of identity and the capacities to clearly communicate this remain intact for PDwD (Sabat and Gladstone, 2010; Castro and Clark-McGhee, 2014).

There is, nonetheless, an implicit understanding in Mick's account that his forgetfulness is a problem, although he does not allude to any diagnosis that might more formally relate to his legal standing. The loss of financial control that he describes demonstrates the influence of the biomedical model within law, positioning him as passive recipient and no longer a citizen with equal rights.

Given the poor reliability and validity of dementia diagnosis (Fox et al., 2013), there are serious ethical concerns regarding diagnosis being linked to legal rights. There are possible connections here to well-documented abuses and failures in care for older adults in the UK (e.g. Equality and Human Rights Commission, 2011; Francis, 2013). Narrative analysis does not allow us to know what has happened to Mick's finances, or how this relates to any capacity decisions, but it does demonstrate how a loss of self-determination threatens Mick's identity construction and, therefore, his well-being.

Mick's construction of his difficulties also hint at how he might wish to be treated; that is, with respect, and with others simply understanding "the fact that the man has a problem". This points to models of dementia care that would enhance his personhood (Kitwood, 1997), with others fully acknowledging both his identity constructions and his rights. 
The tension between protection and inclusion was exemplified by the potential for carers to veto the involvement of relatives who in this research were subsequently assessed to have capacity to consent for themselves. Whilst no vetoes occurred, such would have constituted a form of proxy consent. This is concerning given that, even in the context of applications of the MCA (HMSO, 2005) to the inclusion of people without capacity to consent in research, personal consultees are intended only as sources of advice rather than proxy decisionmakers.

The fact that everyone identified by staff as meeting the inclusion criteria also went on to consent to participate in the research raises questions regarding whether, and how, staff acted as "gatekeepers", in the sense that they may have avoided selecting people with more complex communication needs. Such participants might have raised more difficult questions regarding capacity to consent, and others may have been less amenable to professional requests for participation. Whilst the role of staff to identify and approach potential participants was intended to protect against potential coercion by the researcher, there is also potential for the over-protection of PDwD by carers (paid and unpaid). This has wider implications for the selection of "service user" representatives, and whether people with higher support needs, or those who prove inconvenient in professional systems, for example, by not fulfilling often unacknowledged requirements such as ability to speak articulately, remain unheard.

The ongoing negotiation between ethical practice and pragmatism was recorded in the field diaries. For example, initial discussions with staff about who "usually" consents for themselves involved a distinction between legal relationships and day-to-day relational processes. Next-of-kin arrangements with distant relatives who did not know the participant well were held to be distinct from more relevant, informal day-to-day relationships with staff and carers who regularly support individuals in their decision making. The nature of "usual" decision making was also a source of contention. For example, one participant's wife made decisions related to medication on his behalf, but day-to-day decisions regarding involvement in activities were made by him alone. It is not immediately clear whether participation in research is more akin to the former or the latter. Discussion with both the participant and his wife clarified that, from their perspective, it was a decision he could make alone. Ongoing dialogue with staff, family and the participants, whilst time-consuming, demonstrated the possibilities of making "person-centred" decisions with the person at the centre of the process, as opposed to simply held in mind whilst decisions are made about them by others.

A process model encouraged formal reflection upon these complexities, and is relevant to both care- and research-related decision making, within the guidance of the MCA (HMSO, 2005). In "protecting" people from inclusion, we lose alternative voices which must inform our practices.

\section{Discussion}

The methodological and ethical dilemmas encountered in the course of our research have implications for how we include PDwD more broadly, in that how we position people in research and care contexts reflects and informs broader societal discourses and practices.

\section{Power and "participation"}

This research has been an ongoing process of balancing our longstanding personal and political will to improve the lives of PDwD, with genuinely listening and responding to the particular stories of the participants. Despite our intentions, this endeavour may have 
reproduced existing power relationships through the very act of our researching a group (PDwD) to which we do not belong.

There are many dimensions to this dynamic, most notably related to age, cognitive ability and power (both structural and constructed in interaction). This is exemplified by the narrative of Flo, a participant in her $80 \mathrm{~s}$. The narratives she told of her care were punctuated by remarks in which she questioned the validity of her opinion and expressed a reluctance to criticise care due to a fear of further cuts to her care provision:

Flo: But as I say, I'm very grateful for whatever I am [...] shown [by the carers] to do. Is that, er [...] sensible?

[...]

Flo: Sorry if I'm wrong.

$[\ldots]$

Flo: Well, I mustn't say that [social aspects are more important than healthcare], cos they might stop the healthcare.

Despite assurances of anonymity, what may be Flo's entitlement to criticise, or even comment on, a free service, when it is all that might be available? How entitled were participants to speak openly to a professional introduced to them via a service they receive? There is also a question here around how comfortable people felt to decline participation, either to staff at the initial approach about the research, or when meeting the researcher. It is often a requirement of research ethics committees that researchers are introduced to participants via services, which may link the researcher to a framework of feedback associated with consumerism and service efficiency. This also has implications for service evaluation and indicates a potential tension between policy language emphasising patient choice and control over care (e.g. Department of Health, 2012) when applied to underexplored complexities such as professional-patient dynamics, the potential for coercion and cohort beliefs related to lack of entitlement. Related to this, and as mentioned above, the fact that staff may have acted as gatekeepers, excluding some people from participating in the research, also raises concerns. We approached another voluntary dementia organisation to expand recruitment, and were denied access without further discussion. How can PDwD be central to research processes when professionals exercise their right to determine access? With service providers acting as experts on people defined by "dementia", personhood and citizenship are at stake. PDwD must have authorship of their own lives and identities, and it is incumbent upon professionals in statutory and non-statutory services to facilitate this by developing systems and tools to include and listen to people with a diverse range of abilities and support needs. Otherwise, we risk cherry picking only those able to communicate in forums designed by and for professionals. In regards to our concerns about the potential gatekeeping role of unpaid carers, particularly where people have capacity to make research-related decisions themselves, we stress again the importance of clinical skills to ensure that the voice of the PDwD is elicited and held centrally, that people are engaged in dialogue but not pressured to engage with research (or treatment) and that the dynamics of family and other caring relationships are sensitively attended to.

Service provision for people with learning disabilities, another traditionally-marginalised population, demonstrates a relatively recent history of advancing these agendas in policy and practice (e.g. Department of Health, 2009). In particular, a shift to focus upon the citizenship of people with learning disabilities, inclusion and personalisation, encourages a focus upon rights rather than protection. From our clinical experience working in learning disabilities services, where these policy-driven values are embedded in practice, and prioritised by service leaders, frontline staff have a skilled and nuanced approach to consent and capacity-related decision making. Rather than diagnosis-led decision making, and congruent with the MCA (HMSO, 2005), each decision can be considered with the person with cognitive impairment, who is enabled to participate in the process to the best of their 
ability. This often involves communication aids and, where appropriate, discussion with people who know the decision-maker well.

\section{Participation, compassion and citizenship}

Participatory action research approaches aim for "catalytic validity" (Lather, 1986) in which investigators work with communities from the inception of research through iterative cycles of action and research. We recommend the development of practice-based evidence and participatory research that acknowledge the politics of receiving services and "being researched", and are concerned with democracy, rights and well-being. In a dementia context, this would likely relate to co-construction between people with and without labels of dementia (e.g. Dupuis et al., 2012).

Bartlett and O'Connor (2007) call for dementia care (and by extension, research) that is concerned with equality of rights and compassion, anchored in the recognition that anyone may one day experience similar difficulties. At both the service delivery and policy level, increasing the availability of alternative, personal narratives of dementia and care, may contribute to the re-valuing and inclusion of this population (Castro and Clark-McGhee, 2014). The elicitation and dissemination of narratives of dementia experiences is recommended; for example, locally at multidisciplinary team meetings to plan support, and through wider publication and consideration for policy and guidance. Pragmatism in linking this agenda to mainstream agendas, such as the broader "compassionate care" agenda in the NHS (Francis, 2013), will likely be expedient, and could improve the quality and safety of care by making patient experience central to care.

\section{Future research}

This research demonstrates the possibilities for eliciting and responding to service user views with little resources or power. Process-consent methods constituted good practice guidance for the current research, yet require further development for genuine long-term partnerships in research and action within local contexts. Procedures are required to enable researchers to balance the well-being and dignity of participants, alongside development to fully include traditionally marginalised populations in research, including the engagement of non-English speaking PDwD through suitably trained interpreters or bilingual researchers. Congruent with our approach to analyse the micro and macro narratives within individual stories, no claims are made as to the "representativeness" of our participants in relation to PDwD more broadly. However, future researchers are encouraged to include a more diverse range of participants, given the intersectionality of a host of differences, such as gender, age, ethnicity, sexuality, physical and cognitive ability, in relation to care (Castro Romero, 2016).

In response to the concerns identified regarding the potential gatekeeping role of staff in dementia services it may be advisable for future researchers to build into their study designs recruitment from random sampling of service users. We anticipate that this would result in a reduction in the rate of consent to participate, as there is no preselection for amenability to participation, alongside encountering a more diverse range of communication and other needs. The initial approach of potential participants would still be required by a third party to avoid any possible coercion by the researcher. It may be useful to consider whether the initial approach could be made via trained service user consultants, which may help to address power differentials between carers (paid and unpaid), researchers and PDwD, and the balance between protection and rights.

It is essential that researchers are adequately skilled in the reflexive assessment of consent using verbal and behavioural cues. Based on the ethical dilemmas which arose in this research, we recommend that clinicians trained in research methodology are best placed to 
undertake this sensitive work. It may also be appropriate for clinicians to provide clinical skills training to, and work in advisory and supervisory roles alongside, academic researchers. There are also examples in the literature of recruiting and training researchers to work sensitively with older participants (e.g. Holland, 2005), and possibilities to train people with personal experience of dementia, who often have great skill in supporting and interpreting communication.

Finally, instead of seeking the formal consent of family carers to approach PDwD, it is recommended that researchers foster conversations with all of those involved in the care of PDwD, recording an audit trail of supporting evidence for consent-related decisions. It may be necessary for researchers to advocate for PDwD throughout the process, and challenge gatekeeping where people have capacity to make their own decisions. Researchers may better serve their participants by challenging, perhaps collectively, restrictive ethics requirements that are often designed for medical research rather than soliciting the views of people who may have little other opportunity to author their own lives and identities.

\section{Notes}

1. Our definition of PDwD refers to people who access dementia care services, as the primary service user, i.e. not family, carers, etc. This is congruent with evidence-based guidance that "should not be regarded as applying solely to people with a formal diagnosis of dementia. The support it advocates should be available for all people with cognitive impairment that could be linked to probable dementia" (National Institute for Health and Care Excellence, 2013, p. 1), including mild cognitive impairment.

2. All consultant and participant names are changed for anonymity.

3. Because of ethical requirements, consultants remained in that role and participants were recruited separately.

4. In positioning theory, van Langenhove and Harré (1999) distinguish two aspects of personal identity: the "self" as an enduring worldview which drives one's actions and enables a sense of personal agency, and the "personae" as discursively and publicly produced identity.

\section{References}

Allan, K. (2001), Communication and Consultation: Exploring Ways For Staff to Involve People with Dementia in Delivering Services, Policy Press, Bristol.

Alzheimer's Society (2012), Factsheet 400LP: What is Dementia? Alzheimer's Society, London.

Ayres, L. (2000), "Narratives of caregiving: the process of making meaning", Research in Nursing \& Health, Vol. 23 No. 6, pp. 424-34.

Barnett, E. (2000), Including the Person with Dementia in Designing and Delivering Care. “I Need to be Me!”, Jessica Kingsley, London.

Bartlett, R. and O'Connor, D. (2007), "From personhood to citizenship: broadening the lens for dementia practice and research", Journal of Aging Studies, Vol. 21 No. 2, pp. 107-18.

Behuniak, S. (2010), "Toward a political model of dementia: power as compassionate care", Journal of Aging Studies, Vol. 24 No. 4, pp. 231-40.

Boyle, M. (2003), "The dangers of vulnerability", Clinical Psychology Forum, Vol. 24, pp. 27-30.

Castro, M. and Clark-McGhee, K. (2014), "Poetry written from the words of people given a diagnosis of dementia: a narrative analysis", in Reid, H. and West, L. (Eds), Narratives of Continuity and Change, Routledge, Hove, pp. 144-58.

Castro Romero, M. (2016), "Liberatory praxis alongside elders", in Hughes, G. and Afuape, T. (Eds), Towards Emotional WellBeing Through Liberation Practices: A Dialogical Approach, Routledge, Hove, pp. 127-39.

Clark-McGhee, K. and Castro, M. (2013), "A narrative analysis of poetry written from the words of people given a diagnosis of dementia”, Dementia, Vol. 14 No. 1, pp. 9-26.

Cohen, D. and Eisdorfer, C. (1986), The Loss of Self: A Family Resource for the Care of Alzheimer's Disease and Related Disorders, W.W. Norton, London.

Cowdell, F. (2008), “Engaging older people with dementia in research: myth or possibility?", International Journal of Older People Nursing, Vol. 3 No. 1, pp. 29-34.

Department for Constitutional Affairs (2007), Mental Capacity Act Code of Practice, Department for Constitutional Affairs, London.

Department of Health (2001), National Service Framework for Older People, Department of Health, London.

Department of Health (2009), Valuing People Now: A New Three-Year Strategy for People with Learning Disabilities. Making it Happen for Everyone, Department of Health, London.

Department of Health (2012), Prime Minister's Challenge on Dementia. Delivering Major Improvements in Dementia Care and Research by 2015, Department of Health, London. 
Dewing, J. (2007), "Participatory research: a method for process consent with persons who have dementia", Dementia: The International Journal of Social Research and Practice, Vol. 6 No. 1, pp. 11-25.

Dupuis, S.L., Gillies, J., Carson, J., Whyte, C., Genoe, R., Loiselle, L. and Sadler, L. (2012), "Moving beyond patient and client approaches: mobilizing 'authentic partnerships' in dementia care, support and services", Dementia: The International Journal of Social Research and Practice, Vol. 11 No. 4, pp. 427-52.

Elliott, J. (2005), Using Narrative in Social Research. Qualitative and Quantitative Approaches, Sage, London.

Epp, T.D. (2003), "Person-centred dementia care: a vision to be refined", The Canadian Alzheimer Disease Review, Vol. 5 No. 3, pp. 14-19.

Equality and Human Rights Commission (2011), Close to Home. An Inquiry into Older People and Human Rights in Home Care, Equality and Human Rights Commission, Manchester.

Fox, C., Lafortune, L., Boustani, M. and Brayne, C. (2013), "The pros and cons of early diagnosis in dementia", British Journal of General Practice, Vol. 63 No. 612, pp. 510-12.

Francis, R. (2013), "Report of the Mid Staffordshire NHS Foundation trust public inquiry", executive summary, Stationery Office, London.

Helgesen, A.K., Larsson, M. and Athlin, E. (2010), “Patient participation' in everyday activities in special care units for persons with dementia in Norwegian nursing homes", International Journal of Older People Nursing, Vol. 5 No. 2, pp. $169-78$.

Hernandez, L., Robson, P. and Sampson, A. (2010), "Towards integrated participation: involving seldom-heard users of social care services", British Journal of Social Work, Vol. 40 No. 3, pp. 714-36.

HMSO (2005), Mental Capacity Act (c.9), The Stationary Office Limited, London.

Holland, C. (2005), "Some issues in recruiting and sampling from the older population", in Holland, C. (Ed.), Recruitment and

Sampling: Qualitative Research with Older People, The Representation of Older People in Ageing Research Series, Vol. 5

British Society of Gerontology, York, pp. 1-13.

Hubbard, G., Downs, M. and Tester, S. (2003), "Including older people with dementia in research: challenges and strategies", Aging \& Mental Health, Vol. 7 No. 5, pp. 351-62.

Iphofen, R. (2011), Ethical Decision Making in Social Research, Palgrave Macmillan, Basingstoke.

Katz, J., Holland, C., Peace, S. and Taylor, E. (2011), A Better Life - What Older People with High Support Needs Value, Joseph Rowntree Foundation, York.

Keady, J. and Williams, S. (2007), "Co-constructed inquiry: a new approach to generating, disseminating and discovering knowledge in qualitative research", Quality in Ageing and Older Adults, Vol. 8 No. 2, pp. 27-36.

Kitwood, T. (1997), Dementia Reconsidered: The Person Comes First, Open University Press, Buckingham.

Lather, P. (1986), "Research as praxis", Harvard Educational Review, Vol. 56 No. 3, pp. 257-77.

McCormick, J., Cherti, M., Clifton, J., McDowell, E. and Sachrajda, A. (2009), Getting On: Wellbeing in Later Life, Institute for Public Policy Research, London.

McKeown, J., Clarke, A., Ingleton, C. and Repper, J. (2010), "Actively involving people with dementia in qualitative research", Journal of Clinical Nursing, Vol. 19 Nos 13-14, pp. 1935-43.

Mills, M. (1997), "Narrative identity and dementia: a study of emotion and narrative in older people with dementia", Ageing and Society, Vol. 17 No. 6, pp. 673-98.

National Institute for Health and Care Excellence (2011), Dementia: Supporting People with Dementia and Their Carers in Health and Social Care (Update) (CG042), National Institute for Health and Care Excellence, London.

National Institute for Health and Care Excellence (2013), Quality Standard for Supporting People to Live Well with Dementia, National Institute for Health and Care Excellence, London.

Robson, P., Sampson, A., Dime, N., Hernandez, L. and Litherland, R. (2008), "Seldom-heard: developing inclusive participation in social care", Position Paper No. 10, Social Care Institute for Excellence, London.

Sabat, S.R. and Gladstone, C.M. (2010), "What intact social cognition and social behaviour reveal about cognition in the moderate stages of Alzheimer's disease: a case study", Dementia, Vol. 9 No. 1, pp. 61-78.

Smythe, W.E. and Murray, M.J. (2000), "Owning the story: ethical considerations in narrative research", Ethics and Behaviour, Vol. 10 No. 4, pp. 11-36.

van Langenhove, L. and Harré, R. (1999), "Introducing positioning theory", in Harré, R. and van Langenhove, L. (Eds),

Positioning Theory, Blackwell, Oxford, pp. 14-31.

Wells, D.L. and Dawson, P. (2000), "Description of retained abilities in older persons with dementia", Research in Nursing and Health, Vol. 23 No. 2, pp. 158-68.

Wilkinson, H. (2002), The Perspectives of People with Dementia. Research Methods and Motivations, Jessica Kingsley, London.

\section{About the authors}

Dr Tessa Hughes is a Clinical Psychologist, currently working at the Oldham Memory Assessment and Treatment Service, Pennine Care NHS Foundation Trust, having recently completed her training at the University of East London. Dr Tessa Hughes is the corresponding author and can be contacted at: tessa.hughes@nhs.net

Dr Maria Castro Romero is a Senior Lecturer at the University of East London with a long history as a Clinical Psychologist, Advocate, Researcher, Writer and Trainer, centring on praxis with people with dementia diagnoses to transform the field of "dementia". 